\title{
Opportunities Outside the Traditional Role of Individuals with an Educational Background in Nuclear Medicine Technology and a Bachelor's Degree
}

$\mathbf{T}$ he fact that nuclear medicine is changing is no secret to anyone in this profession. Students and recent graduates have created blogs complaining about the job market, and technologists who are unable to find jobs or have been laid off have fueled the conversation. Educators are seeing decreased enrollment in their programs, and some programs have been closed because of the low number of applicants or financial concerns. Technologists' work hours are being reduced, volume is decreasing in nuclear medicine departments, and equipment is not being replaced as often as in the past. Our elected officers are actively engaged in conversations about the future of the profession and are working to find new opportunities for nuclear medicine. One thing is consistent among all these constituents: they all agree that we live in a new health-care environment. There are two possible views of this scenario-as a glass half full or a glass half empty. I will make the case that the glass is half full and that a multitude of opportunities are available to someone with an educational background in nuclear medicine technology and a bachelor's degree. We have prided ourselves on the fact that nuclear medicine is different and that we possess many skills unique from those of most other allied health professionals. Now is the time to believe this!

For years, many of us have supported and lobbied for a minimum requirement that entry-level technologists have a bachelor's degree. A bachelor's degree is currently only a recommendation and is not mandated or required by the certification bodies or accreditation organizations; however, there are several reasons why this recommendation should become a requirement. In today's competitive world, a bachelor's degree is the standard for many positions within and outside health care. More important, there are many opportunities outside the traditional ones for nuclear medicine technologists.

The more traditional opportunities that for years have been publicized for nuclear medicine technologists still hold true, as do several that may not be as immediately apparent, such as health-care management, education, or

Received Oct. 18, 2013; revision accepted Oct. 24, 2013.

For correspondence or reprints contact: David Gilmore, Regis College, 46 Baldwin St., Boston, MA 02129.

E-mail: david.gilmore@regiscollege.edu

Published online Nov. 14, 2013.

COPYRIGHT (C 2013 by the Society of Nuclear Medicine and Molecular Imaging, Inc.

DOI: $10.2967 /$ jnmt.113.134049 even sales or clinical applications in nuclear medicine. However, there are also other major areas of growth, with multiple jobs, that we should focus on-all of which build on the knowledge and skills acquired in a nuclear medicine program.

The first area of growth is research, or more specifically, the clinical research associate. Many biotech organizations, pharmaceutical companies, universities, and academic medical centers have positions for

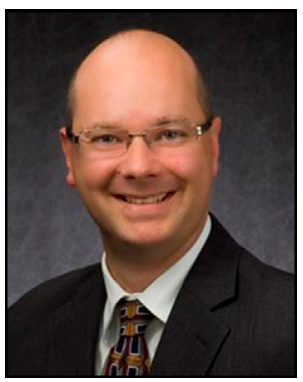
David Gilmore, MS, CNMT, RT(R)(N), FSNMMI-TS compliance of research studies, act as liaisons between groups, implement policies and procedures, and maintain the federal, state, and local files required for research projects. Clinical research associates must be knowledgeable about medical and clinical terminology, be able to complete drug reconciliations and verify protocols, and have the communication and interpersonal skills needed for teamwork and skilled patient care. The one key requirement for these positions is a bachelor's degree in a clinical or science field. There is also typically a certification process, but usually that can be obtained by the hiring organization. For more information on these positions or to see a snapshot of the available positions, visit www.medzilla.com. In looking at the requirements for these positions, it becomes clear that a prepared nuclear medicine technologist could easily fulfill them. Not only do I have previous students in these roles today, but the SNMMI and SNMMI-TS are aggressively working in the area of research and clinical trials. Visit the SNMMI's Web site for more information on the clinical trials network and the educational opportunities it has available.

The next area of growth is health informatics. Because each health-care facility will be required to move to electronic medical records, this field is growing by leaps and bounds. Even without this mandate, technology is advancing the field of informatics for health care. Yes, the individual would need to understand and appreciate the computer technology side of health care, but as nuclear medicine technologists we do possess these skills. The job once thought of for this area is the PACS administrator. Although such positions are still available, there are far greater opportunities 
in the larger arena of health informatics. These positions require an understanding of clinical workflow and healthcare operations, skill in working with physicians and other health professionals, the ability to provide detailed documentation and reporting, and a focus on quality management. Many of these skills are already required in order for nuclear medicine technologists to perform their duties or to complete an accredited nuclear medicine technology program. Visit www.indeed.com and search for health informatics to see the many positions available. Once again, most of these positions require a minimum of a bachelor's degree. There are also opportunities to gain a certificate in health informatics at many colleges and universities.

Finally, I would like to discuss two clinical fields requiring schooling at the master's level or higher in which graduates of a nuclear medicine technology program would do well. Physician assistants and nurse practitioners are both mid-level practitioners for which a graduate of a baccalaureate program would most likely have the prerequisites. Many of the programs for these positions require some clinical background knowledge that is outside an observation. The rigorous clinical internships and competency requirements offered in nuclear medicine typically meet the requirements for such programs; however, it is suggested that schools of interest be viewed and their program admission requirements be evaluated. In the era of the new Affordable Care Act and the Accountable Care Organization model, there is a growing need for these positions.

Although I understand that there may not be a huge number of positions available to the nuclear medicine technologist outside the very traditional one, I do believe that our profession has grown. The education, knowledge, and skills of our profession have allowed us to be competitive in a variety of professions. Here, I have described some areas linked to nuclear medicine, and I have seen my own students obtain some of these positions or move on to other programs; however, I am sure there are many other fields requiring the education we have as nuclear medicine technologists. The key is to be optimistic and willing to grow outside the traditional role to a new role that may or may not yet exist. The idea of lifelong learning has never been more critical than it is now as the landscape of nuclear medicine continues to change. David Gilmore, MS, CNMT, RT(R)(N), FSNMMI-TS
Program Director, Nuclear Medicine Technology
Regis College, Boston, Massachusetts 